\title{
ANÁLISE DE SENSIBILIDADE E CALIBRAÇÃO DO MODELO SWAT APLICADO EM BACIA HIDROGRÁFICA DA REGIÃO SUDESTE DO BRASIL ${ }^{(1)}$
}

\author{
Thatiana Aparecida Lelis ${ }^{(2)}$, Maria Lúcia Calijuri( ${ }^{(3)}$, Aníbal da Fonseca \\ Santiago $^{(4)}$, Dario Cardoso de Lima ${ }^{(5)}$ \& Everton de Oliveira Rocha ${ }^{(6)}$
}

\begin{abstract}
RESUMO
Estimativas da perda de água e solo por erosão têm sido realizadas ao redor do mundo, com base na utilização de modelos empíricos ou conceituais, como o SWAT (Soil and Water Assessment Tool). O SWAT, amplamente utilizado para predizer o impacto das alterações no uso e no manejo do solo, entre outros, sobre a perda de solo e a vazão de curso de água, é extremamente sensível à qualidade dos dados de entrada. Assim, antes da simulação é necessário que se realize uma análise de sensibilidade de tal forma que se possa dar ênfase maior à aquisição e refinamento de determinados dados, diminuir as incertezas e aumentar a confiança nos resultados gerados. $O$ processo de calibração, embora demorado, deve ser sempre realizado a fim de garantir que os resultados da simulação sejam comparáveis aos dados obtidos em campo. $O$ sucesso da aplicação do modelo nessa bacia, sem estudos desse tipo, possibilita que os resultados sejam extrapolados para bacias de características semelhantes. Neste trabalho, a partir dos resultados produzidos em 10 parcelas experimentais instaladas na bacia hidrográfica do ribeirão São Bartolomeu, região Sudeste do Brasil, foram realizadas a análise de sensibilidade e a calibração do modelo SWAT. Os resultados foram satisfatórios, de acordo com o coeficiente de eficiência de Nash e Sutcliffe (COE), utilizado para avaliação do desempenho do modelo, sendo obtidos os valores de COE de 0,808 para a produção de sedimentos e 0,997 para a vazão, os quais representam
\end{abstract}

\footnotetext{
(1) Trabalho extraído de Tese de Doutorado do primeiro autor. Recebido para publicação em 19 de outubro de 2010 e aprovado em 30 de novembro de 2011.

(2) Doutora em Engenharia Civil - Universidade Federal de Viçosa - UFV. Campus Universitário, CEP 36571-000 Viçosa (MG). E-mail: thatianalelis@yahoo.com.br.br

(3) Professora Titular, Universidade Federal de Viçosa - UFV. E-mail: calijuri@ufv.br

(4) Doutorando em Engenharia Civil, Universidade Federal de Viçosa - UFV. E-mail: anibalsantiago@gmail.com

(5) Professor Titular, UFV. E-mail: declima@ufv.br

(6) Mestrando em Engenharia Civil, Universidade Federal de Viçosa - UFV. E-mail: evertonoliveirarocha@hotmail.com
} 
modelos bem calibrados. A análise de sensibilidade não foi influenciada pela maior ou menor discretização da bacia, o que facilitou o processo de análise. A sensibilidade dos parâmetros foi variável em cada sub-bacia, de acordo com seu uso e ocupação, não podendo ser generalizada, isto é, as características das subbacias exercem influência na sensibilidade dos parâmetros.

Termos de indexação: ribeirão São Bartolomeu, sedimentos, escoamento superficial, coeficiente de eficiência.

\title{
SUMMARY: SENSITIVITY ANALYSIS AND CALIBRATION OF SWAT MODEL APPLIED TO A WATERSHEAD IN SOUTHEASTERN BRAZIL
}

\begin{abstract}
Estimates of water and soil losses have been carried out worldwide, using empirical or conceptual models, such as SWAT (Soil and Water Assessment Tool). SWAT, widely used to predict the impact of changes in soil use and management on soil loss and stream flow rate, is extremely sensitive to the quality of input data. Consequently, prior to an application of the model, a sensitivity analysis is required to deal more carefully and with more precision with certain data, reducing the uncertainty and increasing the reliability of the results. Although a slow process, calibration is indispensable to ensure that the simulation results are comparable to the field data. A successful application of the model in this area, with no previous soil and water studies, allows an extrapolation of the results to watersheds with similar characteristics. In this study, a sensitivity analysis and SWAT calibration were carried out with data from 10 plots outlined in the Watershed Sao Bartolomeu, in southeastern Brazil. The results were satisfactory, according to the coefficient of efficiency of Nash and Sutcliffe (COE), used to evaluate the model performance; the COE was 0.808 for sediment yield and 0.997 for the stream flow rate, indicating well-calibrated models. Sensitivity analysis was not influenced by a higher or lower discretization of the watershed, which facilitated the analysis process. The sensitivity of the parameters varied according to the use and soil cover in each sub-watershed, and cannot be generalized, that is, the characteristics of the sub-watersheds influence the parameter sensitivity.
\end{abstract}

Index terms: Sao Bartolomeu river, sediment, runoff, coefficient of efficiency.

\section{INTRODUÇÃO}

A gestão ambiental eficiente dos recursos naturais de uma bacia hidrográfica passa pelo entendimento dos processos que se relacionam direta ou indiretamente com o processo erosivo. De acordo com Paim \& Menezes (2009), a acelerada taxa de erosão do solo é amplamente reconhecida como um problema global, e a avaliação das perdas de solo é de fundamental importância para adoção de práticas que visam minimizar a degradação do solo, uma vez que o uso e a ocupação das terras, juntamente com as características topográficas e climáticas, determinam o balanço sedimentar da bacia hidrográfica.

Dessa forma, é importante o uso de modelos matemáticos para análise e interpretação de informações referentes aos diversos usos do solo e suas consequências para o processo erosivo e o meio ambiente. Além do mais, a análise das interações entre as variáveis condicionantes da erosão dificilmente pode ser feita experimentalmente; em razão disso, a simulação computacional tornase uma forma prática de investigar os impactos provenientes da adoção de diferentes práticas agrícolas. Dessa maneira, o uso de modelos matemáticos e simuladores que representem e integrem as variáveis envolvidas nos processos erosivos apresenta-se eficiente na tarefa de avaliar e entender grande parte dos processos hidrológicos que ocorrem no meio ambiente. Entre esses modelos, destaca-se o "Soil and Water Assessment Tool" SWAT (Srinivasan \& Arnold, 1994; Neitsch et al., 2010).

O SWAT foi desenvolvido para predizer o impacto das alterações no uso, no tipo e no manejo do solo sobre o escoamento superficial e subterrâneo, produção de sedimentos, carga de poluentes e qualidade da água em bacias hidrográficas (Reungsang et al., 2009), sendo amplamente difundido e estudado em todo o mundo (Zhang et al., 2009 e Cibin et al., 2010, nos EUA; Lino et al., 2009 e Baltokoski et al., 2010, na 
América do Sul; Galvan et al., 2007, na Europa; Alansi et al., 2009 e Santosh et al., 2010, na Ásia; e Xie et al., 2010, na África).

\section{Análise de sensibilidade}

O SWAT é extremamente sensível à qualidade dos dados de entrada referentes ao solo. Assim, a acurácia do modelo depende, em grande parte, dos parâmetros que descrevem as características relevantes da bacia hidrográfica e do quão representativos eles são (Adriolo et al., 2008).

Ressalta-se que o SWAT é sensível a uma gama de variáveis de entrada relacionadas a vegetação, manejo e tipo dos solos, clima, aquífero, canal e reservatório (Adriolo et al., 2008; Jha, 2009; Reungsang et al., 2009; Jeong et al., 2010). Devido a esse grande número de parâmetros de entrada, não é possível estudar a incerteza de todos eles. Por isso, é importante que se identifiquem os parâmetros que mais influenciam no modelo e sua importância nos resultados gerados, ou seja, qual a intensidade que cada alteração provoca no modelo, de forma que se possa dar ênfase maior à aquisição e ao refinamento desses parâmetros e, assim, diminuir as incertezas e aumentar a garantia dos resultados gerados (Adriolo et al., 2008; Jha, 2009).
Alguns autores já apresentaram estudos nesse sentido, sendo apontados como os mais sensíveis, na sua maioria, os parâmetros relacionados com as propriedades físicas do solo, como densidade, condutividade hidráulica e, particularmente, capacidade de água disponível, além do número da curva, o qual se relaciona com o solo e a vegetação (Jha, 2009; Cibin et al., 2010). No quadro 1 são apresentados alguns autores e as variáveis identificadas por eles como as mais sensíveis, nas diversas especificidades dos seus trabalhos.

No SWAT 2005, a análise de sensibilidade é realizada pela ferramenta Sensitivity Analysis, que permite identificar quais parâmetros são sensíveis ou os que mais alteram a variável que se deseja calibrar no modelo.

Finalizada a análise de sensibilidade, os parâmetros identificados como os mais sensíveis são quase sempre utilizados para a calibração do modelo (Cibin et al., 2010; Baltokoski et al., 2010). Assim, a análise de sensibilidade deve ser associada à calibração, a fim de se extrair o máximo das potencialidades e recursos do modelo, conduzindo à melhor consistência dos dados gerados e à consequente aplicabilidade na área em estudo ou sua extrapolação para outras áreas semelhantes.

\section{Quadro 1. Parâmetros de maior sensibilidade no SWAT, segundo a literatura}

Número da curva (CN2)

Capacidade de água disponível (SOL_AWC)

Condutividade hidráulica (SOL_K)

Fator de compensação da evaporação do solo (ESCO)

Fator alfa de recessão do fluxo de base (ALPHA_BF)

Profundidade da camada de solo (SOL_Z)

Fração de percolação aquífero profundo(RCHRG_DP)

Fator de cobertura vegetal existente no solo (USLE_C)

Fator práticas de conservação do solo (USLE_P)

Comprimento médio do declive-rampa lateral (SLSUBBSN)

Fator compensação de crescimento da planta (EPCO)

Declividade média da bacia (SLOPE);

Coefic. retardo do escoamento superficial (SURLAG)
Lino et al. (2009); Jha (2009); Reungsang et al. (2009); Cibin et al. (2010); Santosh et al. (2010)

Adriolo et al. (2008); Jha (2009); Schmalz \& Fohrer (2009); Reungsang et al. (2009); Cibin et al. (2010); Jeong et al. (2010); Santosh et al. (2010)

Adriolo et al. (2008); Cibin et al. (2010)

Lino et al. (2009); Jha (2009); Schmalz \& Fohrer (2009); Reungsang et al. (2009); Cibin et al. (2010); Jeong et al. (2010); Santosh et al. (2010)

Lino et al. (2009); Jha (2009); Schmalz \& Fohrer (2009); Reungsang et al. (2009); Cibin et al. (2010); Jeong et al. (2010); Santosh et al. (2010)

Adriolo et al. (2008); Schmalz \& Fohrer (2009)

JhA (2009); Schmalz \& Fohrer (2009)

Adriolo et al. (2008)

Adriolo et al. (2008)

Adriolo et al. (2008); Cibin et al. (2010); Santosh et al. (2010)

Jha (2009); Jeong et al. (2010)

Adriolo et al. (2008); Cibin et al. (2010)

Lino et al. (2009); Cibin et al. (2010); Jeong et al. (2010) 


\section{Calibração e validação}

Devido à variabilidade espacial dos processos simulados pelo SWAT, muitos dos parâmetros de entrada podem não ser exatamente conhecidos. Dessa forma, a calibração é necessária para estimar esses valores ou reduzir sua incerteza, aumentando a confiabilidade dos resultados da modelagem.

O processo de calibração pode ocorrer de maneira manual ou automática. No SWAT 2005 a calibração pode ser feita de forma automática, por meio do recurso autocalibração, conforme método descrito por Mulungu \& Munishi (2007) e van Griensven (2009), baseado na técnica do Shuffled Complex Evolution Algorithm da Universidade do Arizona (SCE-UA). De forma manual ou automática, é usual utilizar métodos estatísticos na etapa de calibração para avaliar o desempenho dos modelos, podendo ser citado o coeficiente de eficiência de Nash e Sutcliffe (COE), conforme utilizaram Jha et al. (2006), Paim $\&$ Menezes (2009) e Ghaffari et al. (2009).

Mesmo sendo um processo demorado e que requer conhecimento amplo do problema, a não execução dessa etapa pode comprometer seriamente todo o resultado obtido por simulação, repercutindo na obtenção de tendências equivocadas (van Griensven, 2009; Zhang et al., 2009).

É importante realçar que a calibração não garante a fidedignidade das predições do modelo. Os parâmetros obtidos do modelo calibrado são tão reais quanto a qualidade e quantidade dos dados fornecidos na calibração. Nesse sentido, Amorim (2004) ressalta que uma série histórica de dados é particularmente importante por causa da variabilidade natural de ano para ano nos fatores que alteram o processo erosivo e o transporte de água e sedimentos; dessa maneira, a imprecisão do modelo pode ser compensada com maior período de tempo.

Uma vez calibrados os parâmetros do modelo, este deve ser validado para situações semelhantes àquelas em que se quer aplicá-lo. Para isso, outra série temporal de dados, diferente da utilizada na calibração, deve ser utilizada no processo de validação (Alansi et al., 2009). Os resultados dessa etapa indicam se o modelo é capaz de reproduzir a série de dados não usados em sua calibração.

O objetivo deste trabalho foi avaliar os parâmetros do SWAT, a fim de definir os mais sensíveis, levandose em consideração a influência do uso e ocupação do solo, topografia e outros fatores físicos e climáticos, variáveis em cada sub-bacia, e, com isso, proceder à calibração e validação do modelo, utilizando como método estatístico o coeficiente de Nash-Sutcliffe (COE) para avaliação dos resultados. Com isso, o sucesso da aplicação desse modelo, numa região em que não há estudos desse tipo, possibilitará que os resultados sejam extrapolados para bacias de características semelhantes.

\section{MATERIAL E MÉTODOS}

\section{Área de estudo}

O presente trabalho foi realizado na bacia hidrográfica do ribeirão São Bartolomeu, localizada entre as coordenadas $20^{\circ} 44^{\prime} \mathrm{S}$ e $42^{\circ} 53^{\prime} \mathrm{O}$, no município de Viçosa, Zona da Mata de Minas Gerais. A bacia ocupa uma área de 5.422 ha, representando cerca de $18 \%$ da área do município, e faz parte da bacia do rio Piranga, a qual é uma sub-bacia do rio Doce, conforme apresentado na figura 1.

A bacia do ribeirão São Bartolomeu, de declividade média igual a $12,8 \%$, tem a maior parte de sua área ocupada por pastagem, somando mais de $38 \%$, distribuída por toda a região; 80 \% da área da bacia pertence às classes de solo Argissolo e Latossolo, conforme trabalho de Lelis \& Calijuri (2010).

De acordo com a classificação de Köppen, o clima da região é do tipo Cwb, ou seja, tropical de altitude com verões frescos e chuvosos (Embrapa, 1982).

\section{Parcelas experimentais}

$\mathrm{Na}$ área de estudo foram instaladas 10 parcelas experimentais, com área de captação de chuva de $40 \mathrm{~m}^{2}$, estrutura coletora de sedimentos e de medição de escoamento superficial, nas quais foi possível o monitoramento do escoamento superficial e das perdas de solo sob condições de chuva natural, durante dois períodos chuvosos (de setembro/2006 a março/2008). A escolha das localidades das parcelas teve como base a declividade das encostas e o tipo de cobertura vegetal, de forma a abranger as principais culturas da bacia.

O escoamento superficial foi determinado a partir de linígrafos instalados em vertedouros de cada parcela. As perdas de solo foram quantificadas por meio da coleta dos sedimentos transportados juntamente com o escoamento superficial até a estrutura de coleta, localizada à jusante de cada parcela experimental, cujo detalhamento do equipamento e do processo de coleta das amostras pode ser visto em Lelis \& Calijuri (2010).

As parcelas experimentais implementadas possibilitaram a medição das perdas de solo e água individualizadas nas culturas predominantes da bacia, sendo admitido que essas parcelas são representativas das condições da região e, consequentemente, da sua perda de solo, que é origem da produção dos sedimentos determinada pelo SWAT. Ressalta-se que, no SWAT, trabalhase com a produção de sedimentos que chega ao canal de fluxo. Essa produção representa a perda de solo (partículas de solo, matéria orgânica, nutrientes, pesticidas, etc.) da bacia ou sub-bacia, sendo, portanto, aplicável a toda a área em estudo, 


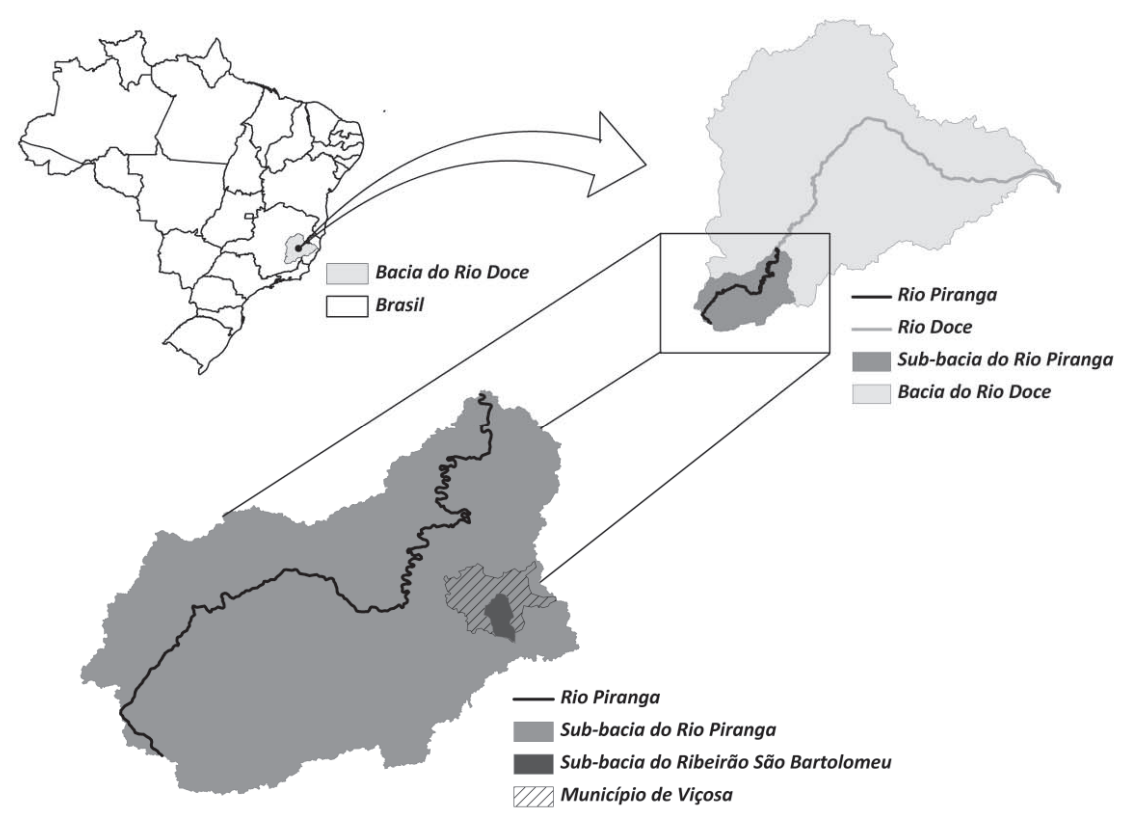

Figura 1. Localização da bacia hidrográfica do ribeirão São Bartolomeu.

admitindo-se a representatividade das parcelas experimentais.

\section{Análise de sensibilidade e calibração do modelo}

Como mencionado, a ferramenta Sensitivity Analysis permite identificar os parâmetros mais sensíveis no SWAT 2005. Segundo van Griensven (2009), dois métodos estatísticos são utilizados no processamento da análise de sensibilidade: Latin Hipercube simulations (LH) e One factor at a time sampling (OAT), os quais são detalhadamente descritos no trabalho de Schmalz \& Fohrer (2009).

Entretanto, a ferramenta Sensitivity Analysis não permite que o usuário defina quais as variáveis a serem analisadas, as quais são definidas (no total de 27) pelo default do modelo (van Griensven, 2009). Como resultado, é gerado um arquivo contendo a posição dos parâmetros analisados,começando de 1 (maior sensibilidade) e podendo chegar até 35 (menor ou nenhuma sensibilidade).

De forma manual, uma maneira de realizar a análise de sensibilidade do modelo variando cada parâmetro de entrada individualmente, enquanto os outros são mantidos constantes, foi apresentada por Silva et al. (2009); esses autores utilizaram o Índice de Sensibilidade Relativo (IS), apresentado a seguir:

$$
\mathrm{IS}=\frac{\frac{\mathrm{R}_{1}-\mathrm{R}_{2}}{\mathrm{R}_{12}}}{\frac{\mathrm{I}_{1}-\mathrm{I}_{2}}{\mathrm{I}_{12}}}
$$

em que IS é o índice de sensibilidade do modelo aos parâmetros de entrada; $R_{1}$, o resultado obtido com o modelo para o menor valor de entrada; $R_{2}$, o resultado obtido com o modelo para o maior valor de entrada; $R_{12}$, a média dos resultados obtidos com o menor e o maior valor de entrada; $\mathrm{I}_{1}$, o menor valor de entrada; $\mathrm{I}_{2}$, o maior valor de entrada; e $\mathrm{I}_{12}$, a média dos valores de entrada.

$\mathrm{Na}$ equação apresentada, quanto maior (em módulo) o índice obtido, mais sensível ao parâmetro será o modelo. Já os valores próximos a zero indicam que o modelo não apresenta sensibilidade ao parâmetro.

Assim, a análise de sensibilidade foi baseada no estudo dos parâmetros e variáveis de entrada do modelo, a fim de indicar aqueles que, quando modificados, influenciam de maneira significativa os resultados. Procurou-se trabalhar com os parâmetros relacionados ao escoamento superficial e à produção de sedimentos, em duas simulações com níveis distintos de discretização da bacia, para estudar, primeiramente, o efeito da maior ou menor subdivisão da bacia na sensibilidade dos parâmetros: simulação 1 - bacia do ribeirão São Bartolomeu (BHRSB) dividida em 25 sub-bacias; e simulação 2 - BHRSB dividida em 392 sub-bacias.

A análise foi inicialmente realizada, de forma automática, por meio da ferramenta Sensitivity Analysis do SWAT 2005, na BHRSB dividida em 25 sub-bacias. Na simulação 1, foram escolhidas quatro sub-bacias distintas e representativas das ocupações predominantes da bacia, conforme 
informações contidas em Lelis \& Calijuri (2010), para análise da sensibilidade dos parâmetros, as quais estão indicadas na figura 2: SB 1 - localizada no estuário da bacia, na zona urbana; SB 12 - situada numa região com predomínio de pastagens não degradadas e mata; SB 17 - em região de mata; e SB 24 - na cabeceira da bacia, ocupada por pastagens degradadas e não degradadas.

A partir dos resultados encontrados nessa primeira simulação, definiram-se os parâmetros que tiveram seus valores alterados no modelo para que se procedesse à análise manual da sensibilidade, conforme a equação 1, variando-se os valores de entrada em $\pm 10 \%$ do valor do parâmetro, conforme recomendações de Jha (2009), Schmalz \& Fohrer (2009) e van Griensven (2009).

Dando continuidade à análise, e com a finalidade de estudar o efeito da maior discretização da bacia na sensibilidade dos parâmetros do modelo, foi realizada a análise automática de sensibilidade com a BHRSB dividida em 392 sub-bacias (simulação 2). Além do mais, esse tipo de tratamento foi importante para conhecer detalhadamente as sub-bacias nas quais se localizavam as parcelas experimentais e os parâmetros que mais influenciariam nos resultados da produção de sedimentos do modelo, especificamente nesses locais.

As sub-bacias escolhidas para análise da simulação 2 tinham as mesmas características

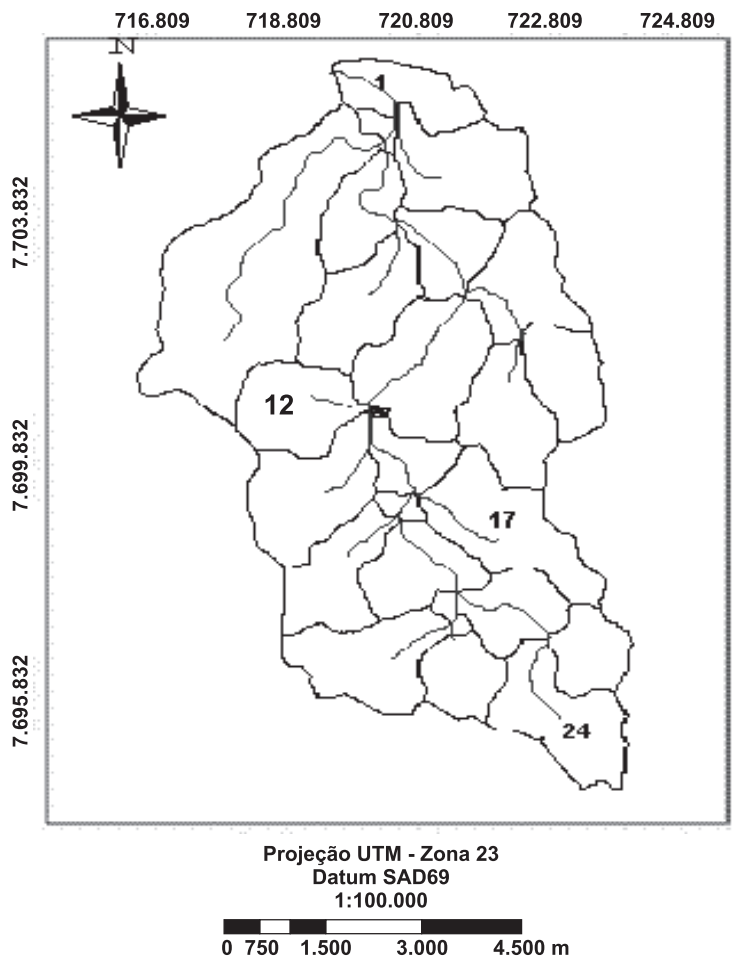

Figura 2. Identificação das sub-bacias objeto da análise de sensibilidade pela simulação 1 . daquelas utilizadas no primeiro estudo de sensibilidade, conforme Lelis \& Calijuri (2010), sendo elas: SB 4, SB 223, SB 278 e SB 387 (Figura 3).

Uma vez identificados na análise de sensibilidade os parâmetros de maior peso nos resultados do modelo, realizou-se a calibração. Para isso, foram utilizados os dados de campo coletados nas parcelas experimentais pelos linígrafos e estruturas coletoras de sedimento, entre janeiro e dezembro de 2007.

A calibração foi iniciada pelo escoamento, que é a parte básica do modelo, e apenas depois do escoamento calibrado prosseguiu-se com a calibração do sedimento, conforme orientações de Adriolo et al. (2008), pois, de maneira geral, os parâmetros de escoamento são dominantes e controlam os resultados da simulação do sedimento e qualidade da água (van Griensven et al., 2005, citados por Adriolo et al., 2008).

A calibração das duas variáveis foi iniciada de forma automática, empregando a ferramenta Autocalibration. No final dessa etapa, de posse dos valores dos parâmetros fornecidos pelo SWAT como os mais adequados para a simulação, eles foram utilizados no modelo para verificar a aproximação com os valores medidos em campo. Em seguida, prosseguiu-se à calibração manual, necessária para fazer ajustes aos parâmetros de entrada do modelo para se chegar a um ajuste satisfatório.

Tanto na forma manual ou automática, para obter-se o ajuste do modelo na calibração, foi

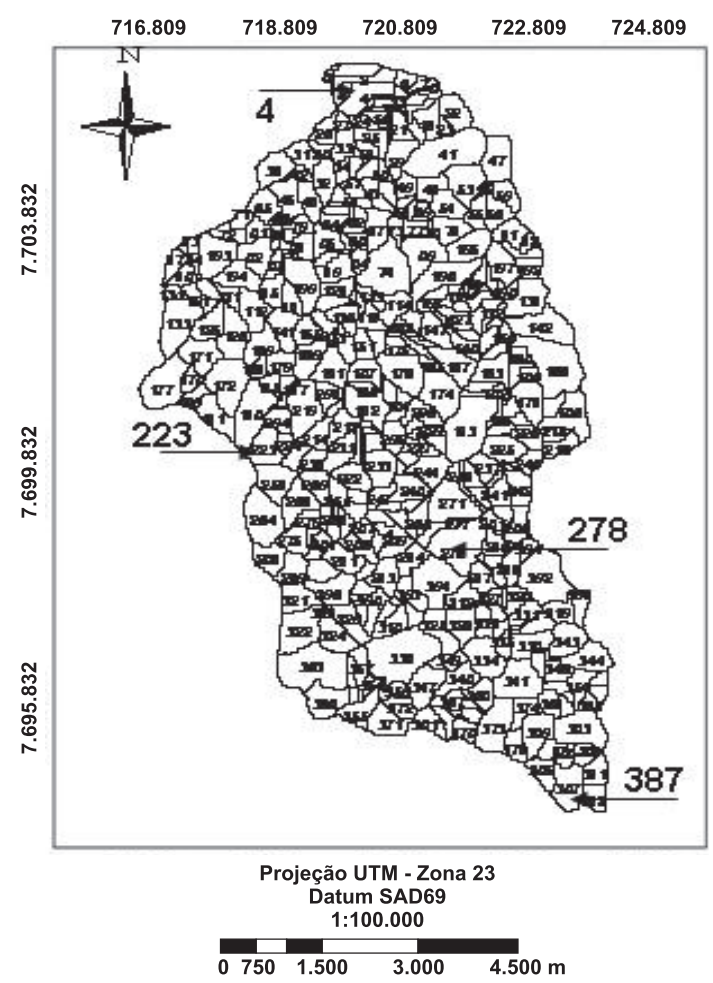

Figura 3. Identificação das sub-bacias objeto da análise de sensibilidade pela simulação 2 . 
utilizado o coeficiente de eficiência de Nash \& Sutcliffe (COE), o qual pode variar de - $\infty$ a 1 , sendo que $\mathrm{COE}=1$ significa ajuste perfeito.

$$
C O E=1-\frac{\sum_{i=1}^{n}\left(E_{o b s}-E_{s}\right)^{2}}{\sum_{i=1}^{n}\left(E_{o b s}-\bar{E}\right)^{2}}
$$

em que $\mathrm{E}_{\mathrm{obs}}$ é o valor do evento observado; $\mathrm{E}_{\mathrm{s}}$, o valor do evento simulado; e E, o valor médio do evento observado.

De acordo com Blainski et al. (2008), para um bom ajuste do modelo são aceitos valores de $\mathrm{COE}$ entre 0,7 e 0,8 , não sendo descartados, entretanto, resultados com COE superior a 0,5. Esse coeficiente de eficiência foi utilizado por Muleta \& Nicklow (2005), Blainski et al. (2008), Baltokoski et al. (2010) e Xie et al. (2010), os quais obtiveram resultados bastante satisfatórios.

Por fim, para confirmar os resultados obtidos pela modelagem, procedeu-se à validação do modelo, empregando dados de perda de solo e água em janeiro de 2008, não utilizados na calibração do modelo.

\section{RESULTADOS E DISCUSSÃO}

No quadro 2 são apresentados os resultados da análise de sensibilidade automática para a variável vazão, realizada pela ferramenta Sensitivity
Analysis do SWAT 2005 para a simulação 1. Nela registram-se o nome do parâmetro no SWAT e o nível de sensibilidade, que variou entre 1 e 28 para a área de estudo nas condições da simulação da bacia dividida em 25 sub-bacias, nas quatro sub-bacias definidas anteriormente.

Os resultados apresentados evidenciam que a sensibilidade dos parâmetros é influenciada, especialmente, pelo uso e ocupação do solo, pela topografia e por outros fatores físicos e climáticos, variáveis em cada sub-bacia (Schmalz \& Fohrer, 2009), conforme o peso de cada um dos fatores. Por exemplo, na sub-bacia 17, de ocupação predominante por mata, o parâmetro de armazenamento de água na copa das árvores (CANMX) foi o mais sensível, enquanto nas outras sub-bacias ele não exerceu tamanha influência.

De modo geral, os resultados foram semelhantes nas quatro sub-bacias; dos parâmetros apresentados, 15 mostraram alguma sensibilidade, ou seja, têm influência sobre o escoamento nas condições estudadas, ressaltando-se que o valor 28 para a sensibilidade significa que o parâmetro não exerce nenhuma influência para a variável analisada. Os resultados apresentados são similares aos obtidos por Mulungu \& Munishi (2007), Schmalz \& Fohrer (2009) e Baltokoski et al. (2010).

Já no quadro 3 são apresentados os resultados da análise automática de sensibilidade, desta vez tanto para a vazão quanto para a produção de sedimentos, com acréscimo de mais seis parâmetros (SPCON - coeficiente linear de fluxo do sedimento; SPEXP

Quadro 2. Análise de sensibilidade automática para a variável vazão - simulação 1

\begin{tabular}{|c|c|c|c|c|}
\hline \multirow{2}{*}{ Nome } & \multicolumn{4}{|c|}{ Ranking } \\
\hline & SB1 & SB12 & SB17 & SB22 \\
\hline ALPHA_BF - Fator alfa de recessão do fluxo de base & 8 & 6 & 6 & 9 \\
\hline BIOMIX - Eficiência de mistura biológica & 13 & 28 & 11 & 14 \\
\hline BLAI - Índice de área foliar potencial máxima & 28 & 28 & 28 & 28 \\
\hline CANMX - Armazenamento de água máximo na copa das árvores & 7 & 4 & 1 & 7 \\
\hline CH_K2 - Condutividade hidráulica efetiva no canal principal & 9 & 7 & 9 & 8 \\
\hline CH_N - Coeficiente de Manning (n) para o canal principal & 11 & 11 & 10 & 12 \\
\hline CN2 - Número da curva & 1 & 3 & 8 & 1 \\
\hline EPCO - Fator de compensação de crescimento da planta & 10 & 10 & 5 & 10 \\
\hline ESCO - Fator de compensação da evaporação do solo & 5 & 5 & 4 & 5 \\
\hline RCHRG_DP - Fração de percolação para o aquífero profundo & 28 & 28 & 28 & 28 \\
\hline SLOPE - Declividade média da bacia & 3 & 12 & 28 & 3 \\
\hline SLSUBBSN - Comprimento médio do declive - rampa lateral & 14 & 28 & 28 & 15 \\
\hline SOL_ALB - Albedo do solo & 15 & 9 & 28 & 13 \\
\hline SOL_AWC - Capacidade de água disponível & 2 & 1 & 2 & 2 \\
\hline SOL_K - Condutividade hidráulica & 4 & 8 & 7 & 4 \\
\hline SOL_Z - Profundidade da camada de solo & 6 & 2 & 3 & 6 \\
\hline SURLAG - Coeficiente de retardo do escoamento superficial & 12 & 13 & 12 & 11 \\
\hline
\end{tabular}


- coeficiente exponencial de fluxo do sedimento; CH_COV - fator de cobertura do canal; CH_EROD - fator de erodibilidade do canal; USLE_C - fator de cobertura vegetal; e USLE_P - fator relativo às práticas de conservação do solo), com níveis variando entre 1 e 35 para as condições da simulação da bacia dividida em 25 sub-bacias, nas quatro sub-bacias anteriormente analisadas.

Com essa combinação de análise, dos parâmetros apresentados, 16 mostraram alguma sensibilidade tanto para a vazão quanto para a produção de sedimentos, e outros três foram sensíveis apenas para os sedimentos produzidos.

Em ambas as análises (Quadros 2 e 3), os parâmetros que se apresentaram mais sensíveis foram o número da curva $(\mathrm{CN})$, a capacidade de água disponível (SOL_AWC), a declividade média (SLOPE), a condutividade hidráulica do solo (SOL_K), a profundidade das camadas do solo (SOL_Z), o fator alfa de recessão do fluxo de base (ALPHA_BF), o fator de compensação da evaporação do solo (ESCO), o armazenamento de água máximo na copa das árvores (CANMX) e, particularmente, o tempo de retardo do escoamento superficial (SURLAG) e o fator de práticas de manejo da USLE (USLE_P) no caso da produção de sedimentos; muitos desses já eram esperados, de acordo com as referências já citadas neste trabalho.

A partir desses resultados, definiram-se os parâmetros que tiveram seus valores alterados no modelo, para que se procedesse à análise manual da sensibilidade, conforme a equação 1. A influência dos parâmetros para as variáveis vazão e sedimentos é apresentada no quadro 4, para as mesmas subbacias analisadas anteriormente. Os parâmetros com valores mais próximos a 1 são os mais sensíveis, estando consoantes com a calibração automática realizada pelo SWAT.

Pelos resultados da calibração manual, os parâmetros mais sensíveis do modelo em relação à vazão foram a profundidade das camadas do solo (SOL_Z), a capacidade de água disponível (SOL_AWC), o fator de compensação da evaporação do solo (ESCO), o fator alfa de recessão do fluxo de base (ALPHA_BF) e o armazenamento de água máximo na copa das árvores (CANMX), todos esses identificados na calibração automática. Entretanto, houve algumas divergências entre os resultados da calibração automática e os da manual, como a grande sensibilidade dos parâmetros GWQMN (profundidade mínima do aquífero superficial para ocorrer escoamento subterrâneo) e BLAI (índice de área foliar potencial máxima) na calibração manual, parâmetros estes identificados como não sensíveis na calibração automática, e a baixa sensibilidade do número da curva $(\mathrm{CN})$ na calibração manual.

Quanto à análise manual da sensibilidade em relação à produção de sedimentos, os parâmetros mais sensíveis foram o número da curva $(\mathrm{CN})$, a declividade média (SLOPE), a capacidade de água disponível (SOL_AWC), a profundidade das camadas do solo (SOL_Z) e o fator de práticas de manejo da USLE (USLE_P), compatíveis com a

Quadro 3. Análise de sensibilidade automática para variáveis vazão e sedimentos - simulação 1

\begin{tabular}{|c|c|c|c|c|c|c|c|c|}
\hline \multirow{3}{*}{ Nome } & \multicolumn{8}{|c|}{ Ranking } \\
\hline & \multicolumn{4}{|c|}{ Vazão } & \multicolumn{4}{|c|}{ Sedimentos } \\
\hline & SB1 & SB12 & SB17 & SB24 & SB1 & SB12 & SB17 & SB24 \\
\hline ALPHA_BF & 1 & 6 & 5 & 8 & 3 & 7 & 8 & 9 \\
\hline BIOMIX & 14 & 13 & 12 & 14 & 6 & 14 & 6 & 16 \\
\hline BLAI & 35 & 35 & 35 & 35 & 35 & 35 & 35 & 35 \\
\hline CANMX & 8 & 4 & 1 & 7 & 10 & 8 & 4 & 11 \\
\hline CH_K2 & 9 & 8 & 9 & 9 & 5 & 10 & 13 & 12 \\
\hline CH_N & 12 & 10 & 10 & 11 & 9 & 12 & 15 & 13 \\
\hline $\mathrm{CN} \overline{2}$ & 2 & 3 & 7 & 1 & 2 & 1 & 1 & 1 \\
\hline EPCO & 10 & 12 & 8 & 10 & 16 & 15 & 11 & 15 \\
\hline ESCO & 7 & 5 & 4 & 5 & 11 & 9 & 10 & 8 \\
\hline RCHRG_DP & 35 & 35 & 35 & 35 & 35 & 35 & 35 & 35 \\
\hline SLOPE & 4 & 14 & 35 & 3 & 8 & 3 & 5 & 4 \\
\hline SLSUBBSN & 15 & 35 & 35 & 15 & 14 & 35 & 35 & 7 \\
\hline SOL_ALB & 13 & 9 & 35 & 13 & 18 & 13 & 14 & 14 \\
\hline SOL_AWC & 3 & 1 & 2 & 2 & 4 & 4 & 7 & 2 \\
\hline SOL_K & 5 & 7 & 6 & 4 & 17 & 11 & 12 & 10 \\
\hline SOL_Z & 6 & 2 & 3 & 6 & 7 & 6 & 3 & 5 \\
\hline SURLAG & 11 & 11 & 11 & 12 & 13 & 2 & 2 & 3 \\
\hline USLE_C & 35 & 35 & 35 & 35 & 19 & $3 \overline{5}$ & $3 \overline{5}$ & 35 \\
\hline SPCON & 35 & 35 & 35 & 35 & 1 & 35 & 35 & 35 \\
\hline SPEXP & 35 & 35 & 35 & 35 & 15 & 35 & 35 & 35 \\
\hline CH_COV & 35 & 35 & 35 & 35 & 35 & 35 & 35 & 35 \\
\hline CH_EROD & 35 & 35 & 35 & 35 & 35 & 35 & 35 & 35 \\
\hline USLE_P & 35 & 35 & 35 & 16 & 12 & 5 & 9 & 6 \\
\hline
\end{tabular}


análise automática, demonstrando a eficiência e confiabilidade dessa ferramenta do SWAT 2005.
Por fim, no quadro 5 são mostrados os resultados de análise de sensibilidade automática para um

Quadro 4. Análise de sensibilidade manual para as variáveis vazão e sedimentos - simulação 1

\begin{tabular}{|c|c|c|c|c|c|c|c|c|}
\hline \multirow{3}{*}{ Nome } & \multicolumn{8}{|c|}{ Ranking } \\
\hline & \multicolumn{4}{|c|}{ Vazão } & \multicolumn{4}{|c|}{ Sedimentos } \\
\hline & SB1 & SB12 & SB17 & SB24 & SB1 & SB12 & SB17 & SB24 \\
\hline ALPHA_BF & 9 & - & 8 & 9 & - & - & - & - \\
\hline BIOMIX & 17 & - & 12 & 16 & - & - & 3 & 8 \\
\hline BLAI & - & 4 & 6 & 5 & - & - & 5 & - \\
\hline CANMX & 7 & 6 & 3 & 15 & 7 & - & - & - \\
\hline CH_K2 & - & - & - & - & - & - & - & - \\
\hline CH_N2 & - & - & - & - & - & - & - & - \\
\hline $\mathrm{CN} 2$ & 5 & 5 & 11 & 14 & 1 & 1 & 1 & 1 \\
\hline EPCO & - & & & - & & - & - & - \\
\hline $\mathrm{ESCO}$ & 6 & 7 & 7 & - & 8 & 9 & - & - \\
\hline GSI & & & & - & - & - & - & - \\
\hline GW_DELAY & 12 & - & 13 & 4 & - & - & - & - \\
\hline GW_REVAP & 11 & - & 9 & 10 & - & - & - & - \\
\hline GWQMN & 13 & 1 & 5 & 8 & - & - & - & - \\
\hline RCHRG_DP & 10 & - & 4 & 3 & - & - & - & - \\
\hline REVAPMN & 15 & - & 10 & 11 & - & - & - & - \\
\hline SLOPE & 4 & - & & 7 & 3 & 3 & 2 & 4 \\
\hline SLSUBBSN & 3 & - & 15 & 6 & 2 & 7 & 7 & 6 \\
\hline SOL_ALB & 14 & 8 & 14 & 13 & - & - & - & - \\
\hline SOL_AWC & 2 & 3 & 2 & 2 & 4 & 2 & - & 3 \\
\hline SOL_K & 8 & 2 & - & 12 & - & 8 & - & - \\
\hline SOL_Z & 1 & - & 1 & 1 & 6 & 4 & - & 2 \\
\hline SURLAG & - & - & - & - & - & - & - & - \\
\hline USLE C & - & - & - & 18 & - & 6 & 6 & 7 \\
\hline USLE_P & 16 & - & - & 17 & 5 & 5 & 4 & 5 \\
\hline
\end{tabular}

Quadro 5. Análise de sensibilidade automática para as variáveis vazão e sedimentos - simulação 2

\begin{tabular}{|c|c|c|c|c|c|c|c|c|}
\hline \multirow{3}{*}{ Nome } & \multicolumn{8}{|c|}{ Ranking } \\
\hline & \multicolumn{4}{|c|}{ Vazão } & \multicolumn{4}{|c|}{ Sedimentos } \\
\hline & SB4 & SB223 & SB278 & SB387 & SB4 & SB223 & SB278 & SB387 \\
\hline ALPHA_BF & 1 & 6 & 2 & 7 & 1 & 7 & 13 & 4 \\
\hline BIOMIX & 14 & 35 & 12 & 12 & 12 & 35 & 8 & 17 \\
\hline BLAI & 35 & 35 & 35 & 35 & 19 & 35 & 35 & 35 \\
\hline CANMX & 6 & 4 & 3 & 8 & 13 & 8 & 4 & 13 \\
\hline CH_K2 & 9 & 8 & 9 & 9 & 4 & 10 & 14 & 6 \\
\hline CH_N2 & 11 & 9 & 10 & 11 & 10 & 14 & 17 & 18 \\
\hline CN2 & 2 & 3 & 8 & 1 & 3 & 1 & 1 & 1 \\
\hline EPCO & 12 & 13 & 11 & 10 & 20 & 35 & 16 & 15 \\
\hline ESCO & 7 & 5 & 6 & 5 & 7 & 9 & 12 & 8 \\
\hline RCHRG_DP & 35 & 35 & 35 & 35 & 35 & 35 & 35 & 35 \\
\hline SLOPE & 5 & 11 & 5 & 3 & 5 & 2 & 5 & 7 \\
\hline SLSUBBSN & 15 & 35 & 13 & 14 & 14 & 35 & 11 & 12 \\
\hline SOL_ALB & 13 & 10 & 14 & 13 & 18 & 12 & 18 & 16 \\
\hline SOL_AWC & 3 & 1 & 1 & 2 & 6 & 4 & 6 & 2 \\
\hline SOL_K & 8 & 7 & 7 & 4 & 16 & 11 & 10 & 11 \\
\hline SOL_Z & 4 & 2 & 4 & 6 & 9 & 6 & 5 & 5 \\
\hline SURLAGG & 10 & 12 & 35 & 15 & 8 & 3 & 7 & 9 \\
\hline USLE_C & 35 & 35 & 35 & 35 & 17 & 35 & 35 & 35 \\
\hline SPCON & 35 & 35 & 35 & 35 & 2 & 35 & 2 & 3 \\
\hline SPEXP & 35 & 35 & 35 & 35 & 15 & 35 & 15 & 14 \\
\hline CH_COV & 35 & 35 & 35 & 35 & 35 & 35 & 35 & 35 \\
\hline CH_EROD & 35 & 35 & 35 & 35 & 35 & 35 & 35 & 35 \\
\hline USLE_P & 35 & 35 & 35 & 35 & 11 & 5 & 9 & 10 \\
\hline
\end{tabular}


cenário mais discretizado da bacia, agora dividida em 392 sub-bacias (simulação 2).

Os resultados mostram que não há influência do tamanho da sub-bacia na sensibilidade dos parâmetros, pois a variação da importância de cada um deles foi muito pequena. A sensibilidade, conforme já visto, é influenciada por fatores físicos e climáticos, variáveis em cada sub-bacia (Schmalz \& Fohrer, 2009). Isso significa que não é necessário um alto nível de discretização da bacia para proceder-se à análise de sensibilidade, o que torna o processo muito mais rápido.

Dessa maneira, a análise manual da sensibilidade no cenário da simulação 2 não foi realizada em razão do grande esforço computacional de se rodar a simulação para tantas sub-bacias várias vezes, do longo tempo a ser despendido e pelo fato de os resultados terem sido compatíveis entre as análises automática e manual da simulação 1 - bacia dividida em 25 sub-bacias e automática da simulação 2, com 392 sub-bacias.

A partir da análise de sensibilidade, foi evidenciado que o peso dos parâmetros é variável em cada sub-bacia, de acordo com seu uso e ocupação, conforme demonstrado nos quadros $2,3,4$ e 5 , isto é, um parâmetro exerce maior ou menor influência no resultado, de acordo com as características da sub-bacia.

Com os resultados obtidos pela análise de sensibilidade, a calibração foi realizada a fim de aferir os valores calculados pelo modelo, utilizando 392 sub-bacias, que foi a maior discretização possível para a bacia. Com essa maior discretização, pretendia-se obter as informações mais precisas e específicas para os locais em que as parcelas experimentais foram instaladas, seguindo, também, a recomendação de Neitsch et al. (2002), que orientaram definir o maior número de sub-bacias para obter-se maior complexidade de uso e tipo de solo ocorrendo na bacia. O quadro 6 apresenta, além dos dados medidos em campo durante o ano de 2007, os valores do modelo sem calibração e o resultado da modelagem após o processo de calibração automática pelo SWAT 2005 e ajuste manual para as variáveis produção de sedimentos e escoamento superficial em algumas sub-bacias da BHRSB. Essas sub-bacias foram escolhidas de modo a representar proporcionalmente todas as combinações de uso e ocupação e tipo do solo definidos no modelo final, com 392 sub-bacias.

Nas duas situações, foi calculado o coeficiente de eficiência (COE), a partir do qual se observou melhora nítida do modelo sem calibração (COE sedimentos $=-152$ e COE escoamento $=-86$ ) em relação à versão final calibrada automática e manualmente (COE sedimentos $=0,808$ e COE escoamento $=0,997)$; os valores finais foram considerados muito satisfatórios, tendo em vista a proximidade dos resultados obtidos com os medidos em campo.

Como mencionado, a calibração não garante a fidedignidade do modelo, porém, pelos resultados apresentados, é indiscutível a melhoria das estimativas após a realização da calibração. No sentido de confirmar os resultados obtidos pela modelagem na bacia do ribeirão São Bartolomeu, procedeu-se à validação do modelo. Os resultados obtidos para o COE na validação, nas mesmas subbacias do quadro 6 , foram de 0,63 para os sedimentos e de 0,85 para o escoamento. Embora tenham sido significantemente inferiores aos alcançados pela modelagem durante o ano de 2007, os valores de COE foram considerados satisfatórios, de acordo com a literatura citada, com a ressalva de que outras pesquisas devem ser feitas para poder consolidar a análise aqui apresentada.

Quadro 6. Resultados do modelo sem calibração e com calibração automática e manual para a produção de sedimentos e escoamento superficial

\begin{tabular}{|c|c|c|c|c|c|c|c|c|}
\hline \multirow{2}{*}{$\begin{array}{c}\text { Sub- } \\
\text { bacia }\end{array}$} & \multirow{2}{*}{$\begin{array}{c}\text { Uso/ } \\
\text { ocupação }\end{array}$} & \multirow{2}{*}{$\begin{array}{c}\text { Classe } \\
\text { solo }\end{array}$} & \multicolumn{3}{|c|}{ Produção de sedimentos (t/ha) } & \multicolumn{3}{|c|}{ Escoamento superficial (mm) } \\
\hline & & & $\begin{array}{l}\text { Perda de } \\
\text { solo média }\end{array}$ & $\begin{array}{c}\text { Sem } \\
\text { calibração }\end{array}$ & $\begin{array}{c}\text { Calibração } \\
\text { SWAT e manual }\end{array}$ & $\begin{array}{c}\text { Escoamento } \\
\text { Superficial médio }\end{array}$ & $\begin{array}{c}\text { Sem } \\
\text { calibração }\end{array}$ & $\begin{array}{c}\text { Calibração } \\
\text { SWAT e manual }\end{array}$ \\
\hline 24 & PSTN & LVA & 0,285 & 0,003 & 0,279 & 118,309 & 472,164 & 117,384 \\
\hline 39 & PSTD & LVA & 0,192 & 4,681 & 0,161 & 2,682 & 586,087 & 8,048 \\
\hline 65 & PSTD & PVA & 0,192 & 0,157 & 0,197 & 2,682 & 434,422 & 10,143 \\
\hline 71 & CAFÉ & CX & 0,008 & 0,000 & 0,000 & 92,604 & 482,009 & 92,648 \\
\hline 92 & MTAL & LVA & 0,015 & 0,436 & 0,015 & 34,307 & 532,979 & 34,086 \\
\hline 95 & PSTD & LVA & 0,192 & 0,176 & 0,153 & 2,682 & 485,906 & 8,045 \\
\hline 97 & MTAL & CX & 0,015 & 0,000 & 0,033 & 34,307 & 481,216 & 33,585 \\
\hline 98 & PSTN & CX & 0,285 & 0,091 & 0,220 & 118,309 & 607,594 & 116,066 \\
\hline 99 & PSTD & $\mathrm{CX}$ & 0,192 & 0,007 & 0,191 & 2,682 & 480,848 & 12,352 \\
\hline
\end{tabular}




\begin{tabular}{|c|c|c|c|c|c|c|c|c|}
\hline \multirow{2}{*}{$\begin{array}{l}\text { Sub- } \\
\text { bacia }\end{array}$} & \multirow{2}{*}{$\begin{array}{c}\text { Uso/ } \\
\text { ocupação }\end{array}$} & \multirow{2}{*}{$\begin{array}{l}\text { Classe } \\
\text { solo }\end{array}$} & \multicolumn{3}{|c|}{ Produção de sedimentos (t/ha) } & \multicolumn{3}{|c|}{ Escoamento superficial (mm) } \\
\hline & & & $\begin{array}{c}\text { Perda de } \\
\text { solo média }\end{array}$ & $\begin{array}{c}\text { Sem } \\
\text { calibração }\end{array}$ & $\begin{array}{c}\text { Calibração } \\
\text { SWAT e manual }\end{array}$ & $\begin{array}{c}\text { Escoamento } \\
\text { Superficial médio }\end{array}$ & $\begin{array}{c}\text { Sem } \\
\text { calibração }\end{array}$ & $\begin{array}{c}\text { Calibração } \\
\text { SWAT e manual }\end{array}$ \\
\hline 101 & MTAL & LVA & 0,015 & 0,393 & 0,016 & 34,307 & 533,981 & 34,088 \\
\hline 112 & PSTN & LVA & 0,285 & 0,003 & 0,303 & 118,309 & 472,164 & 117,379 \\
\hline 122 & PSTN & PVA & 0,285 & 0,020 & 0,188 & 118,309 & 566,130 & 115,872 \\
\hline 183 & MTAL & GX & 0,015 & 0,500 & 0,015 & 34,307 & 450,100 & 37,929 \\
\hline 214 & PSTN & PVA & 0,285 & 0,002 & 0,329 & 118,309 & 424,942 & 115,954 \\
\hline 217 & PSTN & GX & 0,285 & 1,480 & 0,290 & 118,309 & 603,002 & 115,874 \\
\hline 230 & PSTN & LVA & 0,285 & 0,003 & 0,302 & 118,309 & 472,164 & 117,364 \\
\hline 246 & MTAL & $\mathrm{CX}$ & 0,015 & 3,757 & 0,025 & 34,307 & 550,698 & 33,529 \\
\hline 249 & MTAL & PVA & 0,015 & 0,032 & 0,042 & 34,307 & 422,314 & 34,611 \\
\hline 261 & PSTN & PVA & 0,285 & 0,036 & 0,036 & 118,309 & 547,281 & 111,647 \\
\hline 281 & MTAL & LVA & 0,015 & 0,044 & 0,017 & 34,307 & 474,528 & 34,074 \\
\hline 284 & PSTD & LVA & 0,192 & 0,275 & 0,165 & 2,682 & 485,739 & 8,045 \\
\hline 285 & MTAL & PVA & 0,015 & 0,585 & 0,035 & 34,307 & 502,452 & 34,612 \\
\hline 290 & MTAL & LVA & 0,015 & 0,614 & 0,019 & 34,307 & 525,498 & 34,081 \\
\hline 310 & MTAL & PVA & 0,015 & 0,486 & 0,045 & 34,307 & 509,584 & 34,610 \\
\hline 311 & PSTN & PVA & 0,285 & 0,032 & 0,235 & 118,309 & 560,545 & 115,968 \\
\hline 314 & PSTD & PVA & 0,192 & 4,301 & 0,192 & 2,682 & 557,909 & 10,143 \\
\hline 316 & PSTN & LVA & 0,285 & 0,003 & 0,303 & 118,309 & 472,074 & 117,339 \\
\hline 337 & PSTN & CX & 0,285 & 0,000 & 0,291 & 118,309 & 479,067 & 116,078 \\
\hline 350 & PSTD & LVA & 0,192 & 0,147 & 0,168 & 2,682 & 485,805 & 8,045 \\
\hline 370 & PSTD & PVA & 0,192 & 4,020 & 0,188 & 2,682 & 557,976 & 10,140 \\
\hline 376 & CAFE & LVA & 0,008 & 0,370 & 0,000 & 92,604 & 479,984 & 92,364 \\
\hline \multirow[t]{2}{*}{389} & MTAL & LVA & 0,015 & 0,481 & 0,016 & 34,307 & 525,981 & 34,076 \\
\hline & & & $\mathrm{COE}$ & -152 & 0,808 & COE & -86 & 0,997 \\
\hline
\end{tabular}

\section{CONCLUSÕES}

1. Os parâmetros mais sensíveis do modelo foram aqueles relacionados às propriedades físicas do solo e ao manejo, como a capacidade de água disponível, a condutividade hidráulica e o número da curva.

2. A análise de sensibilidade não foi influenciada pela maior ou menor discretização da bacia, o que permitiu agilizar o processo de análise.

3. A sensibilidade dos parâmetros foi variável em cada sub-bacia, de acordo com seu uso e ocupação, não podendo ser generalizada, isto é, as características das sub-bacias exercem influência na sensibilidade dos parâmetros.

4. A calibração gerou resultados compatíveis com os dados de campo, com valores de coeficiente de eficiência (COE) de 0,808 para a produção de sedimentos e de 0,997 para a vazão.

\section{AGRADECIMENTOS}

À FAPEMIG, pelo apoio financeiro, e ao CNPq, pelo projeto $\mathrm{n}^{\circ} 551917 / 2005-9$.

\section{LITERATURA CITADA}

ADRIOLO, M.V.; SANTOS, I.; GIBERTONI, R.C. \& CAMARGO, A.S.G. Calibração do modelo SWAT para a produção e transporte de sedimentos. In: SIMPÓSIO BRASILEIRO DE PEQUENAS E MÉDIAS CENTRAIS HIDRELÉTRICAS, 6., Belo Horizonte, 2008. Anais... Belo Horizonte, 2008.

ALANSI, A.W.; AMIN, M.S.M.; ABDUL HALIM, G.; SHAFRI, H.Z.M. \& AIMRUN, W. Validation of SWAT model for stream flow simulation and forecasting in Upper Bernam humid tropical river basin, Malaysia. Hydrol. Earth System Sci. Discus., 6:7581-7609, 2009. 
AMORIM, R.S.S. Avaliação dos modelos de predição da erosão hídrica USLE, RUSLE e WEPP para condições edafoclimáticas brasileiras. Viçosa, MG, Universidade Federal de Viçosa, 2004. 120p. ( Tese de Doutorado)

BALTOKOSKI, V.; TAVARES, M.H.F.; MACHADO, R.E. \& OLIVEIRA, M.P. Calibração de modelo para a simulação de vazão e de fósforo total nas sub-bacias dos rios Conrado e Pinheiro - Pato Branco (PR). R. Bras. Ci. Solo, 34:253$261,2010$.

BLAINSKI, E.; SILVEIRA, F.A. \& CONCEIÇÃO, G. Utilização do modelo hidrológico SWAT para estudos na microbacia hidrográfica do rio Araranguá/SC. In: TALLER INTERNATIONAL RED RIEGOS - CYTED. Florianópolis, 2008.

CIBIN R.; SUDHEER, K.P. \& CHAUBEY, I. Sensitivity and identifiability of stream flow generation parameters of the SWAT model Hydrol. Process. 2010. Published online in Wiley InterScience (www.interscience.wiley.com) DOI: 10.1002/hyp.7568. p.1133-1148.

EMPRESA BRASILEIRA DE PESQUISA AGROPECUÁRIA EMBRAPA. Atlas climatológico do Estado de Minas Gerais. Belo Horizonte, 1982. 60p.

GALVAN, L.; OLIAS, M.; VILLARAN, R.F. \& SANTOS, J.M.D. Aplicación del modelo hidrológico SWAT a la cuenca del rio Meca (Huelva, España). Geogaceta, 42:63-66, 2007.

GHAFFARI, G.; KEESSTRA, S.; GHODOUSI, J. \& AHMADI, H. SWATsimulated hydrological impact of land-use change in the Zanjanrood Basin, Northwest Iran. Hydrol. Proces., 24:892-903, 2009.

JEONG, J.; KANNAN, N.; ARNOLD, J.; GLICK, R.; GOSSELINK, L. \& SRINIVASAN, R. Development and integration of sub-hourly rainfall-runoff modeling capability within a watershed model. Water Res Manage, 2010. DOI 10.1007/ s11269-010-9670-4.

JHA, M. Hydrologic Simulations of the Maquoketa River Watershed Using SWAT. Center for Agricultural and Rural Development (CARD). Ames, Iowa State University, 2009. (Publications 09-wp492)

JHA, M.; ARNOLD, J.G.; GASSMAN, P.W.; GIORGI, F. \& GU, R. Climate change sensitivity assessment on Upper Mississippi River Basin streamflows using SWAT. J. Am. Water Res. Assoc. 42:997-1016. 2006.

LELIS, T.A. \& CALIJURI, M.L. Modelagem hidrossedimentológica de bacia hidrográfica na região sudeste do Brasil, utilizando o SWAT. R. Amb. Água, 5:158-174, 2010.

LINO, J.F.L.; CORSEUIL, C.W.; KOBIYAMA, M.; MALUTTA, S.; UDA, P.K.; BORTOLOTTO, N.L. \& SANTOS, I. Análise da vazão da bacia hidrográfica do rio Preto com o modelo SWAT. In: SIMPÓSIO BRASILEIRO DE RECURSOS HÍDRICOS, 18., Campo Grande, 2009. Anais... Campo Grande, 2009. 20p.

MULETA, M.K. \& NICKLOW, J.W. Sensitivity and uncertainty analysis coupled with automatic calibration for a distributed watershed model. J. Hydrol., 306:127-145, 2005.
MULUNGU, M.M \& MUNISHI, S.E. Simiyu River catchment parameterization using SWAT model. Phys. Chem. Earth, Parts A/B/C, 32:1032-1039, 2007.

NEITSCH, S.L.; ARNOLD, J.G.; KINIRY, J.R.; SRINIVASAN, R. \& WILLIAMS, J.R. Soil and water assessment user's manual. Version 2000. Grassland, Soil and Water Research Laboratory - Agricultural Research Service e Blackland Research Center - Texas Agricultural Experiment Station, 2002. 412p.

NEITSCH, S.L.; ARNOLD, J.G.; KINIRY, J.R.; SRINIVASAN, R. \& WILLIAMS, J.R. Soil and Water Assessment Input/ Output File Documentation. Version 2009. Grassland, Soil and Water Research Laboratory - Agricultural Research Service, Blackland Research Center - Texas AgriLife Research, 2010. 604p. (Texas Water Resources Technical Report, 365)

PAIM, J.B. \& MENEZES, J.T. Estimativa do balanço sedimentar da bacia do rio Tijucas (SC - Brasil) a partir da aplicação do modelo hidrológico SWAT. R. Geogr. Acad., 3:5-14, 2009.

REUNGSANG, P.; KANWAR, R.S.; JHA, M.; GASSMAN, P.W.; AHMAD, K. \& SALEH, A. Calibration and validation of SWAT for the upper Maquoketa River Watershed. Intern. J. Agric. Eng., 16:35-48, 2009.

SANTOSH, T.; KOLLADI, R. \& SURYA, T. Influence of Scale on SWAT Model Calibration for Streamflow in a River Basin in the Humid Tropics. Water Res. Manage., 2010. Published online: 12/06/2010. DOI 10.1007/s11269-010-9676-y.

SCHMALZ, B. \& FOHRER, N. Comparing model sensitivities of different landscapes using the ecohydrological SWAT model. Adv. Geosci., 21:91-98, 2009.

SILVA, J.M.A.; PRUSKI, F.F.; RODRIGUES, L.N. \& CECILIO, R.A. Modelo para obtenção do hidrograma de escoamento superficial em bacias hidrográficas. R. Bras. Ci. Agr., 4:7884, 2009.

SRINIVASAN, R. \& ARNOLD, J.G. Integration of the basin-scale water quality model with GIS. Water Res. B., 30:453-462, 1994.

van GRIENSVEN, A. Sensitivity, autocalibration, uncertainty and model evaluation in SWAT2005. Artigo técnico. Disponível em: <http://gis-vrac.vrac.iastate.edu/icrw/ download/PrintOut/SWAT2005_manual_sens_cal_unc. pdf. $>$. Acesso em: 10 ago. de 2009.

XIE, H.; NKONYA, E. \& WIELGOSZ, B. Evaluation of the SWAT model in hydrologic modeling of a large watershed in Nigeria. Water Res. Manage., 2010. Africa WRM.

ZHANG, X.; SRINIVASAN, R. \& BOSCH, D. Calibration and uncertainty analysis of the SWAT model using genetic algorithms and bayesian model averaging. J. Hydrol., 374:307-317, 2009. 